\title{
OPEN The transcriptome of Balamuthia mandrillaris trophozoites for structure-guided drug design
}

\author{
Isabelle Q. Phan ${ }^{1,2,12 \bowtie}$, Christopher A. Rice ${ }^{3,11,12 \bowtie}$, Justin Craig ${ }^{1,4}$, Rooksana E. Noorai ${ }^{5}$, \\ Jacquelyn R. McDonald ${ }^{2}$, Sandhya Subramanian ${ }^{1,2}$, Logan Tillery ${ }^{1,4}$, Lynn K. Barrett ${ }^{1,4}$, \\ Vijay Shankar ${ }^{6}$, James C. Morris ${ }^{7}$, Wesley C. Van Voorhis ${ }^{1,4,8,9}$, Dennis E. Kyle ${ }^{3}$ \& \\ Peter J. Myler ${ }^{1,2,9,10 \bowtie}$
}

Balamuthia mandrillaris, a pathogenic free-living amoeba, causes cutaneous skin lesions as well as granulomatous amoebic encephalitis, a 'brain-eating' disease. As with the other known pathogenic free-living amoebas (Naegleria fowleri and Acanthamoeba species), drug discovery efforts to combat Balamuthia infections of the central nervous system are sparse; few targets have been validated or characterized at the molecular level, and little is known about the biochemical pathways necessary for parasite survival. Current treatments of encephalitis due to $B$. mandrillaris lack efficacy, leading to case fatality rates above $90 \%$. Using our recently published methodology to discover potential drugs against pathogenic amoebas, we screened a collection of 85 compounds with known antiparasitic activity and identified 59 compounds that impacted the growth of Balamuthia trophozoites at concentrations below $220 \mu \mathrm{M}$. Since there is no fully annotated genome or proteome of $B$. mandrillaris, we sequenced and assembled its transcriptome from a high-throughput RNA-sequencing (RNA-Seq) experiment and located the coding sequences of the genes potentially targeted by the growth inhibitors from our compound screens. We determined the sequence of 17 of these target genes and obtained expression clones for 15 that we validated by direct sequencing. These will be used in the future in combination with the identified hits in structure guided drug discovery campaigns to develop new approaches for the treatment of Balamuthia infections.

Balamuthia mandrillaris is a ubiquitous soil-dwelling amoeba that is the causative agent of granulomatous amoebic encephalitis (GAE) ${ }^{1-4}$. Similar to the other two major pathogenic free-living amoebas, Naegleria fowleri and Acanthamoeba castellanii, B. mandrillaris infections, though uncommon, have $>90 \%$ case fatality rate ${ }^{5}$. In the United States, 109 Balamuthia cases in both immunocompetent and immunocompromised individuals have been reported with at least twice that many cases worldwide, but these rates of GAE are likely to be underestimated due to historically poor diagnosis ${ }^{6}$. Nonetheless, awareness of potential cases has been on the rise ${ }^{7,8}$. In addition to diagnostic awareness, increasing rates of amoebic infections in northern regions of the United States could also be early indicators that recent emergence of these diseases might be associated with global warming ${ }^{6}$. In contrast to Naegleria infections that present and progress extremely rapidly after exposure, Balamuthia incubation times might be as long as several weeks to months and disease progression more subacute or chronic, increasing the opportunity for therapeutic intervention ${ }^{9}$. However, treatment options remain very limited and not very efficacious, leading to poor outcomes even with the correct diagnosis.

\footnotetext{
${ }^{1}$ Seattle Structural Genomics Center for Infectious Disease (SSGCID), Seattle, WA, USA. ${ }^{2}$ Center for Global Infectious Disease Research, Seattle Children's Research Institute, Seattle, WA, USA. ${ }^{3}$ Center for Tropical and Emerging Global Diseases, University of Georgia, Athens, GA, USA. ${ }^{4}$ Center for Emerging and Re-Emerging Infectious Diseases (CERID), Division of Allergy and Infectious Diseases, Department of Medicine, University of Washington, Seattle, WA, USA. ${ }^{5}$ Clemson University Genomics and Bioinformatics Facility, Clemson University, Clemson, SC, USA. ${ }^{6}$ Center for Human Genetics, Clemson University, Greenwood, SC, USA. ${ }^{7}$ Eukaryotic Pathogens Innovation Center, Department of Genetics and Biochemistry, Clemson University, Clemson, SC, USA. ${ }^{8}$ Department of Microbiology, University of Washington, Seattle, WA, USA. ${ }^{9}$ Department of Global Health, University of Washington, Seattle, WA, USA. ${ }^{10}$ Department of Pediatrics, University of Washington, Seattle, WA, USA. ${ }^{11}$ Present address: Department of Pharmaceutical and Biomedical Sciences, College of Pharmacy, University of Georgia, Athens, GA, USA. ${ }^{12}$ These authors contributed equally: Isabelle Q. Phan and Christopher A. Rice. ${ }^{\square}$ email: isabelle.phan@seattlechildrens.org; christopher.rice@uga.edu; peter.myler@seattlechildrens.org
} 
The recent development of an inexpensive and easily prepared media, as well as increasing interest in B. mandrillaris as a public health concern, has facilitated the development of robust high-throughput drug screening methods. Where low throughput methods restricted screening to $\sim 10-20$ drugs at a time, the new high-throughput methods allow rapid screening of hundreds to thousands of drugs simultaneously and direct comparisons of activity. In this study, we identify FDA approved drugs that could potentially be repurposed for therapy alone or in combination against $B$. mandrillaris. These drugs can also be used as leads for further structure-guided drug discovery (SGDD) exploration and in vivo efficacy studies.

Structure based drug discovery (SBDD) was originally devised in the mid 1980's ${ }^{10}$. Advances in protein structure determination, less expensive and faster computer processing, and better prediction software have reduced the timeline to solve target structures and design specific and selective drugs ${ }^{10}$. SBDD has been mentioned in the amoeba literature as an attractive method to design selective enzymatic inhibitors that specifically target the parasite over the human host ${ }^{11}$, but only a small number of laboratories have actually applied this methodology to pathogenic free-living amoebas. Sterol biosynthesis has been the most attractive target since parasites utilize ergosterol over cholesterol for making cell plasma membranes with distinct host biosynthetic differences that could be selectively targeted ${ }^{12-14}$. Glucose metabolism is essential for parasitic cell viability. Milanes et al., recently targeted glucokinase in N. fowleri, and described NfGlck specific inhibitors with minimal activity against human glucokinase in recombinant enzymatic functional studies ${ }^{15}$. Other studies have looked at targeting histidine or shikimate essential amino acid biosynthetic pathways in Acanthamoeba species, which the hosts cannot synthesize de novo, as parasite specific targets for drug intervention ${ }^{16,17}$.

The development of new compounds against B. mandrillaris in particular has been hampered by the paucity of genomic information. Though draft genomes have been published, no structural and functional annotation is currently available ${ }^{18,19}$. This information is essential for the design of new drugs by SGDD/SBDD, as that methodology requires information about the molecular structure of the target protein. Once the protein coding sequences are annotated on the genome, rapid selection of multiple drug targets can be performed, for example by homology searches with known drug targets, thus paving the way for combinational therapy, a broadly established strategy to minimize the risk of drug resistance. This study presents the first proteome of $B$. mandrillaris reconstructed from RNA sequencing of logarithmic growing trophozoites, the infective form of the amoeba. Potential drug targets identified through phenotypic screening were selected specifically from the trophozoite transcriptome and PCR amplified. The clones were further validated by direct sequencing, providing the first step for recombinant expression and crystallization by the Seattle Structural Genomics Center for Infectious Disease (SSGCID) high-throughput gene-to-structure pipeline ${ }^{20}$.

\section{Results}

Phenotypic screens. We performed a drug susceptibility screen of 85 known anti-parasitic compounds against the trophozoite stage of $B$. mandrillaris and discovered that 59 of these compounds had $50 \%$ inhibitory concentration $\left(\mathrm{IC}_{50}\right.$ ) efficacy at $\leq 220 \mu \mathrm{M}$ concentration (Table 1). Our results indicated that only dequalinium chloride possessed nanomolar potency and that $43 \%$ of the currently recommended drugs by the CDC for treating Balamuthia infections appeared to be only moderately to slightly efficacious, with $\mathrm{IC}_{50}$ values ranging from $18.35 \mu \mathrm{M}$ (pentamidine) to $>163.25 \mu \mathrm{M}$ (fluconazole). Miltefosine, the newest drug addition to the amoebae chemotherapy cocktail, was inactive at the maximum screening concentration of $122.68 \mu \mathrm{M}$. Thus, all currently used therapeutics fall into the unacceptable activity range for today's standards of hit to lead drug discovery and development. Macrolides (azithromycin, clarithromycin, roxithromycin, spiramycin A) have previously demonstrated potent activity against Naegleria or Acanthamoeba but did not show activity against Balamuthia at the concentrations we tested $\left(\mathrm{IC}_{50}\right.$ values $\left.>59 \mu \mathrm{M}\right)$. Although we identified other ribosomal protein inhibitors for 50S (valnemulin, clindamycin, and solithromycin), $23 \mathrm{~S}$ of the $50 \mathrm{~S}$ [mirincamycin (cis- and trans-)], and the $16 \mathrm{~S}$ ribosomal subunits (paromomycin) suggesting protein synthesis would be a useful target for drug therapy against Balamuthia infections. As pentamidine is currently used within the treatment regimen, we screened several anti-protozoal amidines, identifying hexamidine, octamidine and propamidine ( $\mathrm{IC}_{50}$ values $4.46-6.5 \mu \mathrm{M}$ ) as being active against the amoeba. Although the exact mechanisms are still unknown, it is suggested that these compounds interfere with glyceraldehyde 3-phosphate dehydrogenase or interfere with nucleases. We further identified several 3-hydroxy-3-methylglutaryl-Coenzyme A reductase (HMGR) inhibitors (fluvastatin, atorvastatin, and simvastatin), with activity ranging from 1.18 to $3.03 \mu \mathrm{M}$.

Transcriptome sequencing, assembly and functional annotation. Transcriptome assembly and proteome prediction. The sequencing of RNA isolated from an axenic laboratory culture of $B$. mandrillaris trophozoites yielded $30,473,902$ paired-end reads $(2 \times 75 \mathrm{bp})$. We combined three de-novo assemblies, obtained from different assemblers and multiple k-mers, with a genome-based assembly based on the existing B. mandrillaris genome $\mathrm{LFUIO}^{19}$ and then predicted protein coding sequences (CDSs) with EvidentialGene (EviGene) ${ }^{21}$ (Fig. 1).

Of the $37 \mathrm{~K}$ transcripts with predicted CDSs, just over half (53\%) translated to complete proteins. The top 1000 longest complete proteins averaged $1550 \pm 423$ amino acids in length, a number indicative of assembly quality that is roughly comparable to the AmoebaDBv44 A. castellanii proteome $(1688 \pm 539)^{22,23}$. The EviGene "main" sequences, representing the haploid proteome, contained $14 \mathrm{~K}$ proteins; though only $63 \%$ translated to complete proteins, they contain $90 \%$ of complete eukaryotic Benchmarking Universal Single-Copy Orthologs (BUSCOs), a standard measure to quantify the accuracy and completeness of assemblies (Table 2) ${ }^{24}$. Table 2 shows comparable levels of completeness for the transcriptome and the unannotated genome (LFUI01), but the EviGene "main" proteins stand out for containing only 2 duplicates among the complete BUSCOs. 


\begin{tabular}{|c|c|c|c|}
\hline Compound & $\mathrm{IC}_{50}(\mu \mathrm{M}) \pm \mathrm{SEM}$ & Compound & $\mathrm{IC}_{50}(\mu \mathrm{M}) \pm \mathrm{SEM}$ \\
\hline Dequalinium chloride & $0.26 \pm 0.05$ & Pyrimethamine & $52.15 \pm 2.01$ \\
\hline Chlorhexidine & $1.00 \pm 0.09$ & Sitamaquine & $52.45 \pm 2.57$ \\
\hline Fluvastatin sodium & $1.18 \pm 0.24$ & 5-Fluorouracil & $56.63 \pm 2.72$ \\
\hline Atorvastatin & $1.26 \pm 0.25$ & Promethazine & $66.79 \pm 8.93$ \\
\hline HSP990 & $1.80 \pm 0.02$ & Dyclonine HCL & $81.27 \pm 1.51$ \\
\hline Simvastatin & $3.03 \pm 0.31$ & Sulconazole & $81.51 \pm 44.20$ \\
\hline Hexamidine & $4.46 \pm 0.54$ & Dibucaine HCL & $83 \pm 0.65$ \\
\hline WR 99210 & $4.93 \pm 0.08$ & Terbinafine & $83.25 \pm 26.05$ \\
\hline Octamidine & $5.19 \pm 0.01$ & Flucytosine $\Delta$ & $86.34 \pm 17.60$ \\
\hline PHMB & $5.84 \pm 1.60$ & Desipramine & $88.87 \pm 1.67$ \\
\hline Propamidine & $6.50 \pm 0.63$ & Sinefungin & $91.01 \pm 4.83$ \\
\hline Valnemulin & $11.79 \pm 1.12$ & Allopurinol & $92.17 \pm 3.31$ \\
\hline PS-15 (WR 250417) & $14.02 \pm 1.14$ & Floxuridine & $93.18 \pm 3.34$ \\
\hline Benzalkonium chloride & $14.09 \pm 0.07$ & Primaquine & $101.75 \pm 7.94$ \\
\hline Oligomycin B & $14.92 \pm 0.97$ & Tubercidin & $123.17 \pm 0.23$ \\
\hline JPC 2056 & $15.13 \pm 0.43$ & Fluridone & $220.80 \pm 2.87$ \\
\hline Radicicol & $15.22 \pm 1.51$ & Caspofungin & $>45.73$ \\
\hline Trans-mirincamycin & $15.42 \pm 2.84$ & Amphotericin B $\Delta$ & $>54.11$ \\
\hline Mefloquine & $16.54 \pm 3.22$ & Spiramycin A & $>59.31$ \\
\hline Domiphen bromide & $17.08 \pm 0.17$ & Roxithromycin & $>59.73$ \\
\hline Auranofin & $18.24 \pm 0.57$ & Azithromycin & $>66.75$ \\
\hline Pentamidine $\Delta$ & $18.35 \pm 1.47$ & Clarithromycin $\Delta$ & $>66.85$ \\
\hline Cis-Mirincamycin & $18.58 \pm 1.70$ & Natamycin & $>75.11$ \\
\hline Clindamycin & $22.88 \pm 1.05$ & Neomycin & $>81.35$ \\
\hline Chlorpromazine & $24.82 \pm 1.78$ & Tafenoquine succinate & $>85.97$ \\
\hline Solithromycin & $29.66 \pm 0.61$ & Lumefantrine & $>94.53$ \\
\hline Ketoconazole & $29.89 \pm 10.62$ & Verapamil HCL & $>101.82$ \\
\hline Pyronaridine tetraphosphate & $33.56 \pm 1.11$ & Fumagillin & $>109.04$ \\
\hline Amodiaquine & $33.58 \pm 3.46$ & Sertaconazole & $>114.22$ \\
\hline Asenapine & $40.76 \pm 6.16$ & Miltefosine $\Delta$ & $>122.68$ \\
\hline Tioconazole & $42.06 \pm 28.45$ & Atovaquone & $>136.30$ \\
\hline Difenoconazole & $44.92 \pm 19.00$ & Povidone-iodine & $>137.00$ \\
\hline Halofuginone & $46.08 \pm 0.18$ & Voriconazole & $>143.14$ \\
\hline Dihydroartemisinin & $49.06 \pm 2.72$ & Furosemide & $>151.17$ \\
\hline Itraconazole & $49.20 \pm 21.66$ & Quinine & $>154.12$ \\
\hline Posaconazole & $49.57 \pm 12.09$ & Chloroquine & $>156.31$ \\
\hline Paromomycin & $50.03 \pm 0.50$ & Fluconazole $\Delta$ & $>163.25$ \\
\hline Clotrimazole $\Delta$ & $51.53 \pm 23.21$ & Norflurazon & $>164.65$ \\
\hline Climbazole & $51.66 \pm 13.23$ & Chlorpheniramine & $>181.96$ \\
\hline \multirow[t]{2}{*}{ Artesunate $\Delta$} & $52.05 \pm 4.42$ & Proguanil & $>197.06$ \\
\hline & & Glyphosate & $>295.73$ \\
\hline
\end{tabular}

Table 1. Phenotypic analysis of 85 compounds against logarithmic trophozoites in vitro $(\mathrm{N}=2)$. Compounds annotated with $\Delta$ have been previously used to treat GAE or cutaneous Balamuthia infections. The susceptibility is ranked in order of highly potent (left hand side column) to minimal potency (right hand side column) and the inhibitory concentration that causes $50 \%$ ATP depletion (death) is listed as the $\mathrm{IC}_{50} \pm$ standard error mean (SEM). All compounds were initially screened from $50 \mu \mathrm{g} / \mathrm{ml}$ and converted to molarity for standardized testing.

Proteome comparisons. We compared the EviGene 'main' proteins to other species in the UniProt database with AAI-profiler; it retrieved A. castellanii strain Neff as the closest sequenced proteome with $29 \%$ of matched fraction (Fig. 2A). Note that the AAI-profiler does partial sampling as it relies on SANSparallel, a fast homology search that is only as sensitive as BLAST above ca. $50 \%$ sequence identity ${ }^{25}$. We confirmed this result with a BLASTP search of the EviGene 'main' proteins against $A$. castellanii: it returned hits for $65 \%$ of the Balamuthia sequences, with an average identity of $44 \%$, but matched $29 \%$ with at least $50 \%$ identity.

To gain further insight on how closely the two proteomes are related, we performed orthologous cluster analysis with Dictyostelium discoideum as the outgroup. Results show that $38 \%$ of the Balamuthia proteins cluster with 


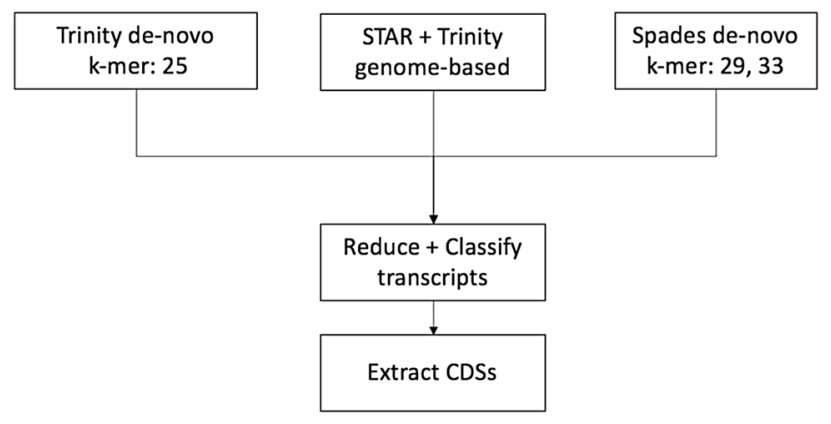

Figure 1. Overview of the main steps for predicting the B. mandrillaris proteome from RNA-seq reads using a hybrid approach. De-novo and genome-based assemblies are combined and processed with EviGene to reduce transcript redundancy and classify transcripts as encoding complete or incomplete protein coding sequences (CDSs, $5^{\prime}$ and/or 3' truncated). CDSs are extracted, translated and annotated as "main" or alternate.

\begin{tabular}{|l|l|l|l|l|l|l|}
\hline & \multicolumn{2}{|l|}{ Assembled transcriptome } & EviGene "main" proteins & \multicolumn{2}{l|}{ Genome (LFUI01) } \\
\hline \# Input sequences & 37,076 & 14,255 & \multicolumn{2}{l|}{1605} \\
\hline Complete BUSCOs & 287 & $95 \%$ & 270 & $89 \%$ & 271 & $89 \%$ \\
\hline Single-copy & 86 & $28 \%$ & 268 & $88 \%$ & 166 & $55 \%$ \\
\hline Duplicated & 201 & $66 \%$ & 2 & $1 \%$ & 105 & $35 \%$ \\
\hline Fragmented BUSCOs & 4 & $1 \%$ & 6 & $2 \%$ & 10 & $3 \%$ \\
\hline Missing BUSCOs & 12 & $4 \%$ & 27 & $9 \%$ & 22 & $7 \%$ \\
\hline
\end{tabular}

Table 2. BUSCO quality and completeness assessment of the B. mandrillaris EviGene transcript assembly and predicted proteome compared to the draft genome (reference dataset for eukaryotes: $\mathrm{N}=303$ ). Italic values describe Complete BUSCOs (single-copy + duplicated).

Acanthamoeba, of which $21 \%$ are shared between the three species (Fig. 2B). This result and the high proportion of singletons (48\%) highlights the divergence of Balamuthia from Acanthamoeba.

To place the Balamuthia proteome in an evolutionary context, we constructed a neighbor-joining tree from an alignment-free comparison of complete proteomes from selected Amoebas, with the non-Amoebozoa Naegleria as outgroup (Fig. 2C). As detected by AAI-profiler, the Discosea genera Balamuthia and Acanthamoeba are in a separate group from the Variosea genus Planoprotostelium and the Eumycetozoa Dictyostelids Cavenderia, Polysphondylium, Tieghemostelium and Dictyostelium. The Evosea genus Entamoeba is in a separate branch from the other Amoebozoa in the tree.

Functional annotation of the draft proteome. To characterize the proteome further and expand the pool of potential targets, we conducted preliminary functional annotations of the draft proteome dataset. Functional annotation of the EviGene "main" protein sequences with PANNZER2, one of the top-10 rapid methods in the CAFA2 NK-full benchmark, provided $26 \%$ of the sequences with a description and $25 \%$ with a lower level GO molecular function term. A plot of high-level GO terms compared with those obtained for A. castellanii and D. discoideum, one of the most thoroughly annotated amoebas in UniProt, shows a similar profile for the three species, with differences limited to smaller gene families representing less than $1 \%$ of the genes (Fig. 3 ). In terms of potential targets for SBDD, the Balamuthia GO annotations classified $284(2 \%)$ of proteins as having kinase activity, of which over half (163) were classified as protein kinases, but further analysis is needed to target the kinome with accuracy ${ }^{26}$.

Target identification and validation. For this study, putative protein kinase targets from the phenotypic screens were left out, leaving a total of 25 potential targets (out of the original list of 52), to which we added 6 potential drug targets requested by the amoeba community via the gene-to-structure service portal of the SSGCID website. From this list of 31 protein names, 19 could be assigned to specific human protein sequences. A total of 14 Balamuthia sequences for 13 targets (there are 2 copies of topoisomerase II) were identified from a BLASTP search with the human sequences: 12 from the phenotypic screens, and 2 community targets. Average pairwise identity was $49 \%$ with $77 \%$ coverage. Another three of the community targets were not detected by BLASTP searches of the Balamuthia proteome using the human sequences, therefore Acanthamoeba sequences were used instead. This yielded a total of 17 Balamuthia sequences that were entered into the SSGCID gene-to-structure pipeline. Truncations around putative catalytic domains were designed for 9 of the 17 sequences to increase crystallization likelihood, leading to 23 constructs as cloning candidates. PCR amplification produced clones for 18 constructs. Direct sequencing was successful for 15 of these and sequence comparison with the "main" proteins from the EviGene assembly showed excellent matches with over $99 \%$ average amino acid identity, corresponding 


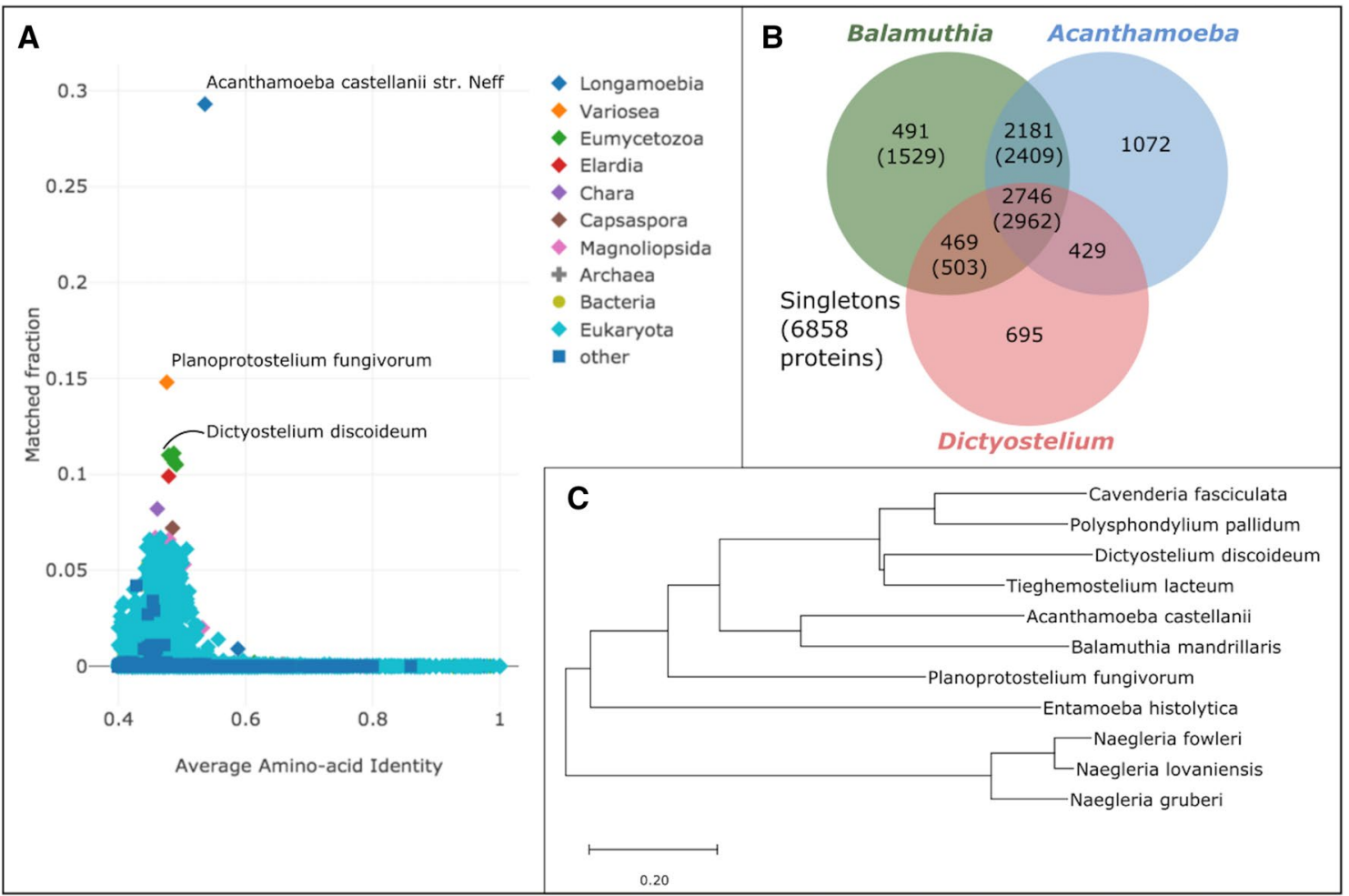

Figure 2. (A) AAI-profiler scatterplot. Shown are UniProt species with greater than $40 \%$ average amino-acid identity to the Balamuthia 'main' proteins. The species name of the top three proteomes with the largest fraction of matches to Balamuthia are indicated. (B) Venn diagram showing the overlap between orthologous cluster groups in the proteomes of $B$. mandrillaris, A. castellanii and D. discoideum. Total numbers of $B$. mandrillaris proteins in each group are in parenthesis. (C) Neighbor-joining tree illustrating the phylogenetic relationships between the proteomes of $B$. mandrillaris and closest species. The tree is based on alignment-free comparisons of the closest complete proteomes detected by AAI-profiler with three Naegleria species as outgroups.

to 2 amino acid variations on average per sequence, and 100\% coverage for all but the two largest proteins (84\% coverage and 100\% identity for the 1068 amino-acid long Exportin-1, 81\% coverage and 99\% identity for the 784-residue primase and C-term domains of topoisomerase II).

The identity between the 15 validated protein sequences and their closest $A$. castellanii homologue ranged between 56 to $88 \%$, with 3 notable exceptions: exportin-1 (21\% identity), lanosterol 14-alpha demethylase (CYP51A) (28\% identity) and glucokinase (51\% identity). In the case of exportin-1, a multiple sequence alignment indicated that the Balamuthia protein was over $50 \%$ identical to the $N$. fowleri and Planoprotostelium fungivorum proteins, suggesting potential mis-assembly in the A. castellanii genome. Similarly, the Acanthamoeba glucokinase sequence appears to have a large deletion of over 30 residues compared to the Balamuthia and Naegleria sequences. This region corresponds to a double-stranded beta-sheet that lies in the glucose binding pocket in the Naegleria structure, and we would expect it to be conserved in Acanthamoeba (Fig. 4$)^{15}$.

Of the 13 targets that were also found in humans, five shared over 55\% sequence identity overall to their human counterpart and might potentially have similar active sites: $S$-adenosyl-homocysteinase (SAHH), 3-hydroxy-3-methylglutaryl-coenzyme A reductase (HMGR), heat-shock protein 90-alpha (HSP90a), histone deacetylase 1 (HDAC1) and exportin-1 (XPO1) (Table 3). As a consequence, SBDD for these targets will likely require exploration of potential alternate binding sites that are specific to the Balamuthia protein. We would expect selectivity to be more readily achievable for the other targets, with the topoisomerase ATPases as borderline cases. One promising example of a Balamuthia target that can be selectively targeted is the GARTFase domain of trifunctional purine biosynthetic protein adenosine-3 (GART). Balamuthia GARTFase has a low sequence identity to the human enzyme (37\%) and has a different domain arrangement than in human GART. Whereas GARTFase is the C-terminal domain of human GART, it is the middle domain in Balamuthia (Fig. 5). This domain arrangement, confirmed by direct sequencing and conserved in Acanthamoeba, leads us to postulate that targeting double domains in GART may offer a promising avenue to develop drugs against those pathogenic amoebas.

\section{Discussion}

Based on previously determined in vitro activity and the few surviving cases of Balamuthia GAE infections, the Centers for Disease Control and Prevention (CDC) recommends that the drug cocktail regimen for treating disease include a combination of pentamidine, sulfadiazine, flucytosine, fluconazole, azithromycin or clarithromycin, and miltefosine ${ }^{6}$. 

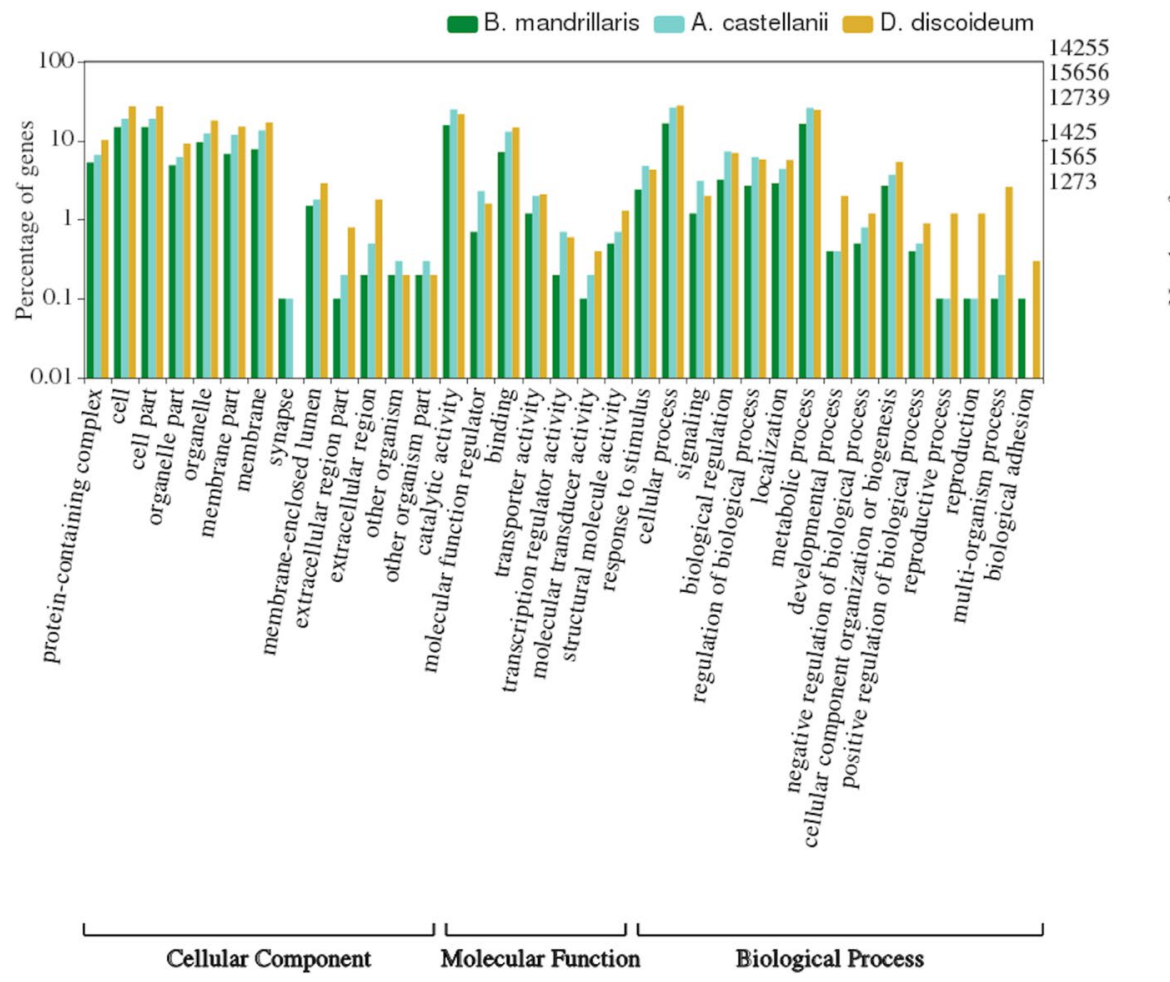

Figure 3. Level 2 top GO annotations. B. mandrillaris proteins (dark green) vs A. castellanii (cyan) and D. discoideum (yellow) as percentage of genes and total number of genes on a $\log (10)$ scale, significant relationships p-value $<0.05$.
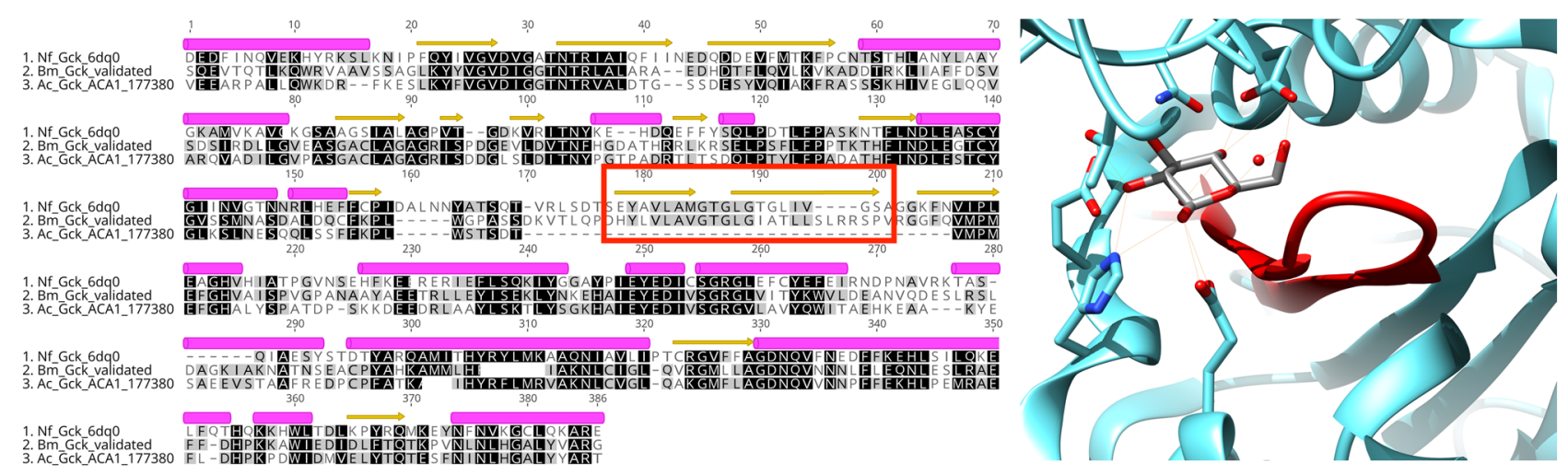

Figure 4. Sequence conservation of glucokinase in 3 pathogenic amoebas. Potential mis-assembly of the $A$. castellanii glucokinase (AmoebaDB ACA1_177380) highlighted on a multiple sequence alignment with the B. mandrillaris validated sequence (this study) and $N$. fowleri crystal structure (PDB: 6DA0) ${ }^{15}$; helical regions are annotated as pink tubes and beta-sheets as yellow arrows. The alignment was obtained with T-CoffeeExpresso $^{27}$. The double-stranded beta-sheet missing in A. castellanii glucokinase is colored in red on the active site of the B. mandrillaris structure (PDB: 6VZZ).

We thus proceeded to test these compounds first in order to confirm inhibition and assess if they have sufficient potency $(<10 \mu \mathrm{M})$ for hit-to-lead phenotypic drug screening. According to our screens, none of the recommended compounds passed this criterion. We therefore expanded our drug screening to other compounds that previously displayed anti-Acanthamoeba, anti-Naegleria, or anti-malarial activity, starting with the macrolides.

Our screening results consistently indicate that the compounds belonging to the macrolide drug class (azithromycin, clarithromycin, roxithromycin, and spiramycin) are inactive, in agreement with previous results ${ }^{28}$. Interestingly, solithromycin, a known ketolide antibiotic against macrolide-resistant Streptococcal species ${ }^{29}$, appeared to show moderate activity against $B$. mandrillaris $(29.66 \mu \mathrm{m})$. We found that polyene antimycotics such as amphotericin B and natamycin, that target ergosterol within fungal cell membranes ${ }^{30}$, also were also inactive against $B$. mandrillaris. We then tested the azole compounds. CDC studies reported that fluconazole 


\begin{tabular}{|c|c|c|c|}
\hline Balamuthia target & Pairwise identity & Target coverage & Closest human protein \\
\hline S-adenosyl-L-homocysteine hydrolase ${ }^{\mathrm{b}}$ & $58 \%$ & $98 \%$ & sp|P23526|SAHH_HUMAN \\
\hline Histone deacetylase 1 & $73 \%$ & $83 \%$ & \begin{tabular}{|l} 
sp|Q13547|HDAC1_HUMAN \\
\end{tabular} \\
\hline Lanosterol 14-alpha demethylase (CYP51A) & $26 \%$ & $96 \%$ & sp|Q16850|CP51A_HUMAN \\
\hline $\begin{array}{l}\text { Methionyl-tRNA synthetase (methionine tRNA ligase) } \\
\text { (MetRS) }\end{array}$ & $54 \%$ & $79 \%$ & sp|P56192|SYMC_HUMAN \\
\hline Heat shock protein HSP90-alpha & $69 \%$ & $100 \%$ & \begin{tabular}{|l|l|} 
sp|P07900|HS90A_HUMAN \\
\end{tabular} \\
\hline Calcium ATPase, haloacid dehydrogenase (HAD) domain & $43 \%$ & $100 \%$ & tr|A0A0A0MSP0|ATP2C2_HUMAN \\
\hline $\begin{array}{l}\text { 3-Hydroxy-3-methylglutaryl-CoA reductase (HMG-CoA } \\
\text { reductase) (HMGR) }\end{array}$ & $62 \%$ & $97 \%$ & sp|P04035|HMDH_HUMAN \\
\hline Glucokinase $^{\mathrm{b}}$ & - & - & None \\
\hline $\begin{array}{l}\text { DNA topoisomerase II copy 1, ATPase and transducer } \\
\text { domains }\end{array}$ & $55 \%$ & $97 \%$ & sp|Q02880|TOP2B_HUMAN \\
\hline DNA topoisomerase II copy 1 , toprim and C-term domains & $49 \%$ & $99 \%$ & \begin{tabular}{|l} 
sp|P11388|TOP2A_HUMAN \\
\end{tabular} \\
\hline $\begin{array}{l}\text { DNA topoisomerase II copy 2, ATPase and transducer } \\
\text { domains }\end{array}$ & $52 \%$ & $97 \%$ & sp|P11388|TOP2A_HUMAN \\
\hline DNA topoisomerase II copy 2, toprim and C-term domains & $39 \%$ & $80 \%^{\mathrm{a}}$ & \begin{tabular}{|l} 
sp|Q02880|TOP2B_HUMAN \\
\end{tabular} \\
\hline Exportin-1 (CRM1, XPO1) & $57 \%$ & $83 \%^{\mathrm{a}}$ & sp|O14980|XPO1_HUMAN \\
\hline Xylose isomerase $(x y l A)^{b}$ & - & - & none \\
\hline $\begin{array}{l}\text { Trifunctional purine biosynthetic protein adenosine-3 } \\
\text { (GART), GARTFase domain }\end{array}$ & $37 \%$ & $82 \%$ & sp|P22102|PUR2_HUMAN \\
\hline
\end{tabular}

Table 3. Sequence similarity (BLASTP) between Balamuthia validated sequences and UniProt identifiers of closest human counterpart. ${ }^{a}$ Lower than expected coverage due to incomplete sequencing of the clones. ${ }^{b}$ Additional targets selected by the community. Note that homology to human Glucokinase was too low to be detected with BLASTP at the chosen E-value (1e-3).
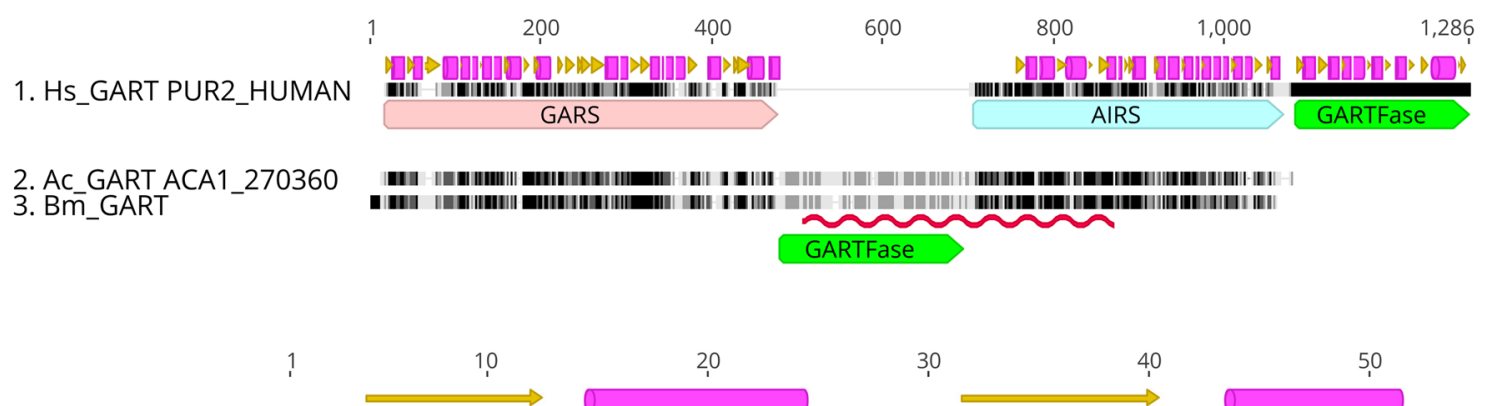

1. Hs GARTFase

2. AC GARTFase

3. Bm_GARTFase

ARVAVL I ISGTGSNL QAL IDSTREP N SISAQ|DIVI SNK AAVA GLDKAE RAGI NNTLRLAVLGSTRGTDMOA I IDA I ARRE LNAF I ALVVSNRADAY ILERARAHGI TAPTR | GILGSTRGTDMQA I IDA I EQGK LINAEVKIMVVSNVEDAY ILERAR L HNL 60

1. Hs GARTFase 2. AC GARTFase

3. Bm_GARTFase

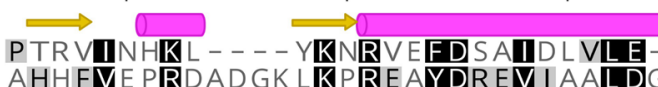
- -EF SIDIVCLAGFMR ILSGPFVQK VIDGGVDLVL M IGYMR IVSAAFVER PHTFLPSIKG - - - - KKRETFDKQVVQILD- - EAGVELVLL IGFMR ILSPVFVRH

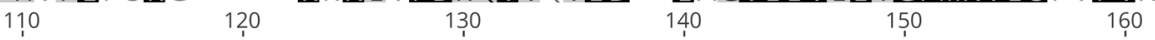

1. Hs GARTFase

2. Ac_GARTFase

3. Bm_GARTFase

1. Hs GARTFase

2. Ac_GARTFase

3. Bm_GARTFase

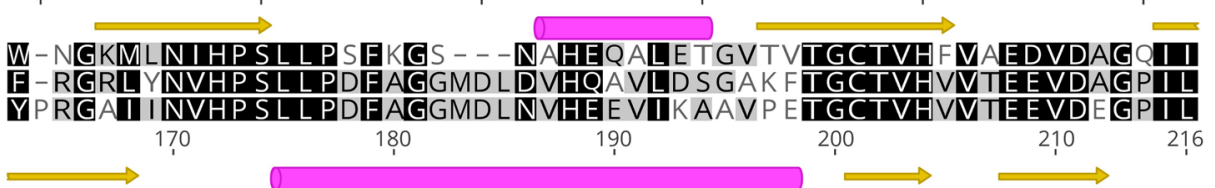

Figure 5. Top: Domain arrangement in human, A. castellanii and B. mandrillaris GART. Domains are annotated as large arrows on the alignment and higher level of residue conservation is represented as darker shades of gray. The region validated by direct sequencing in Balamuthia is underlined with a red squiggle. Secondary structure elements from human GART crystal structures are taken from UniProt. Bottom: Alignment of the GARTFase domains extracted from the GART sequences above. 
was inactive at concentrations lower than $10 \mu \mathrm{g} / \mathrm{mL}^{31}$. We were able to confirm the fluconazole result; however, other antifungal azoles (ketoconazole, tioconazole, difenoconazole, itraconazole, posaconazole, clotrimazole, climbazole, and sulconazole) displayed better, though still moderate, activity $(29.89-81.51 \mu \mathrm{m})$. We tested flucytosine and miltefosine and both displayed moderate to poor activity against $B$. mandrillaris with an $\mathrm{IC}_{50}$ of 86.34 and $>122 \mu \mathrm{m}$, respectively. Flucytosine was previously described to inhibit $61 \%$ Balamuthia cytopathogenicity at $10 \mu \mathrm{g} / \mathrm{mL}(77 \mu \mathrm{m})$. Combined with our own results, this suggests flucytosine and miltefosine are equipotent ${ }^{32}$. Although miltefosine has been reported to have moderate activity at concentrations of $63-100 \mu \mathrm{m}^{32,33}$, another study $^{34}$ agrees with our data that miltefosine is less potent with a higher $\mathrm{IC}_{50}$ value $>122 \mu \mathrm{m}$. As for pentamidine, our results are consistent with prior studies that obtained comparable IC $_{50}$ values between 9 and $29 \mu \mathrm{m}^{31,35,36}$.

Thus, of the combination drug cocktail recommended by the CDC, only pentamidine and flucytosine appear to have in vitro activity against $B$. mandrillaris. It is possible that the recommended drug therapy is active in combination, and not when tested in isolation as in this study. It is also possible that the drugs are biologically activated in vivo or are only active against an in vivo form that we have not assayed. We therefore cannot rule out activity for the recommended drugs based solely on our in vitro sensitivity screens. Known inhibitors of $50 \mathrm{~S}$ and $16 \mathrm{~S}$ ribosomal proteins, such as macrolides and lincosamides, showed no or very poor activity in our screens, but we can only speculate that translation in Balamuthia evolved independently of prokaryotic endosymbiosis. Though Parachlamydia acanthamoebae was shown to infect $B$. mandrillaris ${ }^{37}$, we could find no evidence of $P$. acanthamoebae $50 \mathrm{~S}$ ribosomal proteins in our de-novo transcriptome assembly.

We previously identified statins, which target 3-hydroxy-3-methylglutaryl-coenzyme A reductase (HMGR), as active compounds against $B$. mandrillaris ${ }^{36}$. As part of this study, we tested three additional statins: fluvastatin, atorvastatin and simvastatin and observed that they were active against B. mandrillaris at $1.18-3.03 \mu \mathrm{M}$ concentration. Simvastatin and fluvastatin in particular have been shown to have better brain penetration compared to other statins ${ }^{38}$, indicating potential for drug repurposing. These results not only validate this drug class as promising lead molecules for the potential treatment of $B$. mandrillaris GAE, but also HMGR as a priority target of interest for future studies.

From a drug-repurposing standpoint, the compounds described in this study yielded a plethora of potentially useful drugs that act on $B$. mandrillaris. Available chemical inference data associated only few of the compounds with a specific protein target. Given our low success rate of structure determination in parasites, we increased the pool of potential protein targets by including results from our previous drug discovery studies. These include screening the MMV Malaria and Pathogen boxes that identified 11 compounds with equipotency to nitroxolone (8-Hydroxy-5-nitroquinoline) $\mathrm{IC}_{50}$ of $2.84 \mu \mathrm{M}^{32}$, indicating these molecules could be used as an initial starting point for medicinal chemistry structure activity relationship (SAR) studies. More recently, we identified 63 compounds through screening the Calibr ReFRAME drug repurposing library, with activities ranging from $40 \mathrm{nM}$ to $4.8 \mu \mathrm{M}^{36}$.

Chemical inference from our previous drug screening efforts and the results from this study identified a total of 52 potential protein targets in B. mandrillaris. After excluding kinases, our target list was reduced to 25 proteins. Of these, 15 were identified solely from this study and the remainder was identified in several of our screening efforts. These 25 protein targets and an additional 6 community targets of interest were submitted for structural determination to the Seattle Structural Genomics Center for Infectious Diseases (SSGCID) gene-tostructure pipeline.

The first step of the SSGCID pipeline involves cloning of the B. mandrillaris sequences encoding the protein targets identified in the screens. However, lack of annotation of the Balamuthia genome hampered these efforts: although we were able to locate some sequences using BLAST searches of the Balamuthia genome using the human and Acanthamoeba homologues, PCR amplification from B. mandrillaris cDNA (or gDNA) was not successful. Therefore, transcriptome sequencing of Balamuthia was performed.

We reconstructed a draft $B$. mandrillaris proteome from a transcriptome that we obtained by combining 4 different assemblies from a single RNA-seq experiment. According to the BUSCO standard benchmark, our set of Evigene 'main' proteins is as complete (90\%) as the reference genome, but with only a fraction of duplicates. We have thus based our subsequent analysis on this set as a representative of the haploid proteome of $B$. mandrillaris trophozoites. From our search of UniProt complete proteomes, A. castellanii stood out as the closest species with no other close relative, making the Acanthamoeba and Balamuthia proteomes the sole representatives of the phylum Discosea. As a third of the B. mandrillaris proteins appeared to be truncated, our phylogenetic analysis is based on an alignment-free method suitable for computing evolutionary distances between complete or incomplete proteomes ${ }^{39}$. The evolutionary tree placed the Discosea in a separate group as expected, highlighting their degree of divergence to the other pathogenic amoeba $N$. fowleri. Although they belong to the same phylum, Acanthamoeba and Balamuthia appear to be distantly related according to our alignment-based analyses-less than half of the proteins clustered together, and just above a quarter shared over $50 \%$ sequence identity.

We obtained functional annotation for above a quarter of the proteins using a conservative method. Annotation with the less stringent blast2go still leaves $60 \%$ of sequences with no annotation, a $20 \%$ higher proportion than the Acanthamoeba proteome. Much work remains to be done to characterize the complete proteome. Nonetheless, validation through direct sequencing showed that for our chosen targets at least, our assembly is accurate. In addition, alignment of the validated sequences to the genome revealed frameshifts in the reference assembly, which was further supported by analyzing the genome annotation we obtained with GENSAS ${ }^{40}$. Our attempts to correct the genome by re-assembling the publicly available PACBIO reads with modern assemblers and correcting exons with the transcripts were unsuccessful. Future efforts might succeed once the 454 Illumina reads used in the published genomic assembly will be made accessible.

The majority of the protein targets that we identified in Balamuthia had closely related sequence homologues in Acanthamoeba, a likely indication that they perform essential function in both organisms. From the high level of sequence identity, we would expect similar pattern of inhibition with the drugs tested in this report. However, 
our preliminary studies indicate that this is not the case. For example, the heat shock protein HSP90 is highly conserved in Balamuthia and Acanthamoeba (78\% pairwise sequence identity), and yet we measured up to 5.56fold differences in $\mathrm{IC}_{50}$ values in response to the same drug. Another example is 3-hydroxy-3-methylglutaryl-CoA reductase; the sequence identity is still high at $70 \%$, but when we compared responses to nine different statins, we observed large differences ranging from $>2.51$ to $>112$-fold differences. The final example is lanosterol 14-alpha demethylase which displayed the least pairwise identity to A. castellanii protein sequence with $28.5 \%$ similarity. B. mandrillaris was found to be less susceptible to the 11 different azoles tested with 0.79 to 646 -fold difference compared to the response of $A$. castellanii. The implication for drug discovery is that we cannot assume that orthologous sequences in Balamuthia and Acanthamoeba will be close enough to permit simultaneous targeting of these two pathogenic amoebas.

\section{Conclusion}

Through drug susceptibility screening with known antiparasitic compounds against B. mandrillaris, we identified protein targets with potential for treating Balamuthia granulomatous amoebic encephalitis. The reconstruction and annotation of the draft proteome from RNA-seq allowed us to amplify, clone and validate the B. mandrillaris targets by direct sequencing.

The haploid proteome appears distantly related to that of its closest relative A. castellanii, and thus provides an essential resource for further drug discovery and biological investigation. Even in cases where the target sequences are conserved, our preliminary results indicate that potent inhibitors against Acanthamoeba or Naegleria failed to inhibit Balamuthia growth, suggesting that the quest for a broad-spectrum drug against all three pathogenic amoebas might prove elusive. Drugs will need to be specifically developed for each amoeba.

This study illustrates how the combination of phenotypic drug screening and a single RNA-seq experiment with short reads are enabling structure-based drug design against a eukaryotic pathogen with no prior proteome information. As there are currently no genetic tools available for B. mandrillaris, the results presented here will enable future studies to validate specific drug targets. Validation strategies may include co-crystallization of protein and inhibitors; development of drug resistant $B$. mandrillaris clones followed by whole genome sequencing; specifically targeting genes through RNAi (which has been used successfully in other free-living amoebae) or, in the era of CRISPR/Cas9-mediated gene editing, the generation of conditional knock outs, would be some promising ways of target validation for future studies within this pathogen.

\section{Materials and methods}

Cell culture. Maintenance of Balamuthia mandrillaris. The pathogenic B. mandrillaris (CDC:V039; ATCC 50209), a GAE isolate, isolated from a pregnant Baboon at the San Diego Zoo in 1986 was donated by Luis Fernando Lares-Jiménez ITSON University, Mexico ${ }^{35}$. Trophozoites were routinely grown axenically in BMI media at $37{ }^{\circ} \mathrm{C}, 5 \% \mathrm{CO}_{2}$ in vented $75 \mathrm{~cm}^{2}$ tissue culture flasks (Olympus), until the cells were $80-90 \%$ confluent. For sub-culturing, $0.25 \%$ Trypsin-EDTA (Gibco) cell detachment reagent was used to detach the cells from the culture flasks. The cells were collected by centrifugation at $4000 \mathrm{rpm}$ at $4{ }^{\circ} \mathrm{C}$. Complete BMI media is produced by the addition of $10 \%$ fetal bovine serum and $125 \mu \mathrm{g}$ of penicillin/streptomycin antibiotics. All experiments were performed using logarithmic phase trophozoites.

Target identification. Phenotypic screening. We previously developed and standardized robust highthroughput screening methods for the discovery of active compounds against B. mandrillaris trophozoites ${ }^{35}$. The trophocidal activity of compounds were assessed using the CellTiter-Glo 2.0 luminescent cell viability assay (Promega, Madison, WI). In brief, B. mandrillaris trophozoites cultured in BMI-complete media were seeded at 16,000 cells/well into 96-well plates (Thermo Fisher 136102) with various compounds diluted in twofold serial dilutions to determine the $50 \%$ inhibitory concentration $\left(\mathrm{IC}_{50}\right)$. The highest percentage of DMSO diluted in the highest screening drug concentration was $1 \%$. Control wells were supplemented with $1 \%$ DMSO or $12.5 \mu \mathrm{M}$ of chlorhexidine, as negative and positive controls, respectively. All assays were incubated at $37^{\circ} \mathrm{C}$ for $72 \mathrm{~h}$. At the end time point, $25 \mu \mathrm{L}$ of CellTiter-Glo reagent was added to all wells. The plates were shaken using an orbital shaker at $300 \mathrm{rpm}$ at room temperature for $2 \mathrm{~min}$ to induce cell lysis. After shaking, the plates were equilibrated at room temperature for $10 \mathrm{~min}$ to stabilize the luminescent signal. The ATP luminescent signal (relative light units; RLUs) were measured at $490 \mathrm{~nm}$ by using a SpectraMax i3X (Molecular Devices, Sunnyvale, CA). Drug inhibitory concentration $\left(\mathrm{IC}_{50}\right.$ ) curves were generated using total ATP RLUs where controls were calculated as the average of replicates using the Levenberg-Marquardt algorithm, using DMSO as the normalization control, as defined in CDD Vault (Burlingame, CA, USA). Values reported are from a minimum of two biological replicates with standard error of the mean.

Selection of target genes. The protein names for verified potential targets were retrieved through Calibr at Scripps Research (https://reframedb.org/). The corresponding human protein sequences were downloaded from UniProt and queried against the $B$. mandrillaris assemblies using BLAST sequence similarity searches. Candidate targets were confirmed by comparing their protein sequences with closest sequence homologues in Acanthamoeba and Naegleria species and checking the B. mandrillaris functional annotation, where available. ORFs were selected for cloning from the Trinity de-novo assembly. Manual correction of putative start sites from multiple sequence alignments was performed with Geneious Prime 2019.1.1 (https://www.geneious.com).

RNA extraction, library preparation and sequencing. RNA extraction. Balamuthia mandrillaris were cultured and harvested as described above; the cells were counted and adjusted to 2 million cells for each extraction. Total RNA isolated using the RNA extraction kit (Agilent) as per manufacturing instructions. In 
brief, to the pellet of Balamuthia cells, $350 \mu \mathrm{L}$ of lysis buffer and $2.5 \mu \mathrm{L}$ of $\beta$-mercaptoethanol were added and homogenized. This was transferred into a prefilter spin cup and centrifuged at maximum speed, $14,000 \times g$, for $5 \mathrm{~min}$. The filtrate was retained and an equal volume of $70 \%$ ethanol was added to the filtrate and vortexed until the filtrate and ethanol were mixed thoroughly. This mixture was then transferred into an RNA binding spin cup and receptacle tube and centrifuged at maximum speed for $1 \mathrm{~min}$. The filtrate was discarded and 600 $\mu \mathrm{L}$ of $1 \times$ low salt buffer was added and centrifuged at maximum speed for $1 \mathrm{~min}$. The filtrate was removed and centrifuged at maximum speed for $2 \mathrm{~min}$. DNase solution was added and incubated for $15 \mathrm{~min}$ at $37^{\circ} \mathrm{C}$. After incubation $600 \mu \mathrm{L}$ of $1 \times$ high salt buffer (contains guanidine thiocyanate) was added and centrifuged at maximum speed for $1 \mathrm{~min}$. The filtrate was discarded and $300 \mu \mathrm{L}$ of $1 \times$ low salt buffer was added and centrifuged at maximum speed for $2 \mathrm{~min}$. $100 \mu \mathrm{L}$ of elution buffer was added and incubated at room temperature for $2 \mathrm{~min}$. Final elution was into a sterile $1.5 \mathrm{~mL}$ microcentrifuge tube at maximum speed for $1 \mathrm{~min}$.

Extracted RNA was stored at $-80^{\circ} \mathrm{C}$ until further required. The integrity and purity of the RNA was assessed via RT-PCR and gel electrophoresis on a $2 \%$ agarose gel. The concentration was determined by measuring $280 \mathrm{nM}$ absorbance on a nanodrop (Nanodrop 1000, Thermo Scientific).

RNA quality was reassessed after a freeze thaw cycle using the Bioanalyzer RNA 6000 pico chip (Agilent, 50671513 ) and quantity was assessed using the Qubit RNA Broad Range Assay (Invitrogen, Q10210). The mRNA was isolated using the NEB Poly(A) mRNA Magnetic Isolation Module (NEB, E7490S) and prepared using a version of the Stranded RNA-seq protocol that was modified for Leishmania ${ }^{41,42}$. Only the negative stranded RNA-seq library preparation portion was performed. Library quantity and quality was assessed using the Qubit dsDNA High Sensitivity Assay (Invitrogen, Q32851), Bioanalyzer High Sensitivity DNA Chip (Agilent, 5067-4627) and the KAPA library quantification kit (Roche, KK4824). Libraries were sequenced on the Illumina Hiseq 4000, yielding $2 \times 75$ bp paired end reads.

Transcripts assembly and annotation. Reads were quality filtered with Trimmomatic and assembled de-novo with Trinity v2.8 $(\mathrm{k}$-mer $=25)$ and Spades v3.13 $(\mathrm{k}$-mer $=29$ and 33) after clipping of the adaptor sequences ${ }^{43-45}$. Further, quality-filtered reads were aligned to the published B. mandrillaris genome LFUI01 with STAR v2.6 and assembled with Trinity ${ }^{46}$. The three assemblies thus obtained were combined with EvidentialGene v19jan01 (EviGene) with BUSCO homology scores as input for the classifier ${ }^{21}$. Throughout the analysis, BUSCO v3 analysis was performed on either the European or Australian Galaxy mirrors ${ }^{47,48}$. Assemblies were screened for vector and common eukaryotic contaminants with the EvidentialGene utilities asmrna_vecscreen and asmrna_trimvec. Functional descriptions and gene ontology (GO) annotations of the EviGene 'main' proteins were predicted with PANNZER2 ${ }^{49}$. GO annotations that were highest ranked by PANNZER2 were visualized with WEGO $2.0^{50}$.

Comparison to other species and phylogenetic analysis. Proteome comparisons to other species in the UniProt database were obtained from the AAI-profiler server ${ }^{51}$. Cluster analysis and Venn diagrams of orthologous clusters were generated with OrthoVenn $2^{52}$ (e-value: $1 \mathrm{e}-5$, inflation value: 1.5$)$. Unless otherwise specified, all BLAST searches were performed with BLAST + v2.8.1 and an expectation value of $0.001^{53}$. Pairwise distances for alignment-free phylogeny reconstruction were calculated with Prot-SpaM ${ }^{39}$. Input sequences included the Balamuthia EviGene 'main' proteins (this study), AmoebaDB A. castellanii strain Neff, N. fowleri ATCC 30894 and N. lovaniensis Braker1 predicted proteins ${ }^{54,55}$, and UniProt complete reference proteomes $(C$. fasciculata UP000007797, N. gruberi UP000006671, P. pallidum UP000001396, D. discoideum UP000002195, $P$. fungivorum UP000241769 and T. lacteum UP000076078). Phylogenetic relationships were inferred by constructing a neighbor-joining tree from the word match-based Prot-SpaM distance matrix using MEGA X ${ }^{56,57}$.

PCR and sequence validation. Cloning. All B. mandrillaris constructs were cloned, expressed, and purified using SSGCID established protocols ${ }^{58,59}$. The genes selected were PCR-amplified using cDNA template and purchased primers (Integrated DNA Technologies, Inc., Coralville, IA) (Supplementary Table S1). The amplicons were extracted, purified and cloned into a ligation-independent cloning pET-14b derived, N-terminal His tag expression vector $\mathrm{pBG} 1861$ with a T7 promoter ${ }^{60}$. The cloned inserts were then transformed into purchased GC-5 cells (Genesee Scientific, El Cajon, CA) for ORF incorporation. Plasmid DNA was purified from the subsequent colonies and further transformed in chemically competent E. coli BL-21(DE3)R3 Rosetta cells with a chloramphenicol restriction.

Sequence validation. Each B. mandrillaris construct was sequenced from both $5^{\prime}$ - and $3^{\prime}$-ends with a custom forward primer (5'-GCGTCCGGCGTAGAGGATC-3', 40nt upstream from the T7 promoter customary forward primer) and the T7 terminator reverse primer (5'-GCTAGTTATTGCTCAGCGG-3') at GeneWiz (South Plainfield, NJ). The reads were assembled and matched to the expected sequences with the phrap assembler and cross_match ${ }^{61}$. Translations of the longest ORF in all six frames of the consensus sequence (or the forward read if unassembled) were then aligned using MUSCLE ${ }^{62}$ with the SSGCID target protein sequence to determine the best translated protein sequence and its alignment, percent identity and percent coverage. Manual examination of the sequences and alignments was performed in Geneious.

\section{Data availability}

Illumina raw reads have been deposited at the National Center for Biotechnology Information (NCBI) BioProject repository with the accession number SRR12006108 under project PRJNA638697. This Transcriptome Shotgun Assembly project has been deposited at DDBJ/EMBL/GenBank under the accession GISS00000000. The version described in this paper is the first version, GISS01000000. The annotated protein sequences from the EviGene 
'main' assembly are available on NIH Figshare under https://doi.org/10.35092/yhjc.12478733.v1. All data that are associated with the drug susceptibility study are archived using the database from Collaborative Drug Discovery (CDD; http://www.collaborativedrug.com/). The CDD database accommodates both compound chemistry data and results from phenotypic or target activity, cytotoxicity screening, and computed properties. The CDD database is becoming an established standard for the sharing of data within this community, and we are eager to facilitate the distribution of our results in a similar manner.

Received: 29 May 2021; Accepted: 27 September 2021

Published online: 04 November 2021

\section{References}

1. Visvesvara, G. S., Schuster, F. L. \& Martinez, A. J. Balamuthia mandrillaris, N. G., N. Sp., agent of amebic meningoencephalitis in humans and other animals. J. Eukaryot. Microbiol. 40, 504-514 (1993).

2. Schuster, F. L. et al. Environmental isolation of Balamuthia mandrillaris associated with a case of amebic encephalitis. J. Clin. Microbiol. 41, 3175 (2003).

3. Cabello-Vílchez, A. M. et al. The isolation of Balamuthia mandrillaris from environmental sources from Peru. Parasitol. Res. 113, 2509-2513 (2014).

4. Niyyati, M., Karamati, S. A., Lorenzo Morales, J. \& Lasjerdi, Z. Isolation of Balamuthia mandrillaris from soil samples in NorthWestern Iran. Parasitol. Res. 115, 541-545 (2016).

5. Gompf, S. G. \& Garcia, C. Lethal encounters: The evolving spectrum of amoebic meningoencephalitis. IDCases 15, e00524 (2019).

6. Cope, J. R. et al. The epidemiology and clinical features of Balamuthia mandrillaris disease in the United States, 1974-2016. Clin. Infect. Dis. 68, 1815-1822 (2019).

7. Shehab, K. W., Aboul-Nasr, K. \& Elliott, S. P. Balamuthia mandrillaris granulomatous amebic encephalitis with renal dissemination in a previously healthy child: Case report and review of the pediatric literature. J. Pediatr. Infect. Dis. Soc. 7, e163-e168 (2018).

8. Yang, Y., Hu, X., Min, L., Dong, X. \& Guan, Y. Balamuthia mandrillaris-related primary amoebic encephalitis in China diagnosed by next generation sequencing and a review of the literature. Lab. Med. https://doi.org/10.1093/labmed/lmz079 (2019).

9. Pritzker, A. S., Kim, B. K., Agrawal, D., Southern, P. M. \& Pandya, A. G. Fatal granulomatous amebic encephalitis caused by Balamuthia mandrillaris presenting as a skin lesion. J. Am. Acad. Dermatol. 50, S38-41 (2004).

10. Anderson, A. C. The process of structure-based drug design. Chem. Biol. 10, 787-797 (2003).

11. Byington, C. L., Dunbrack, R. L., Whitby, F. G., Cohen, F. E. \& Agabian, N. Entamoeba histolytica: Computer-assisted modeling of phosphofructokinase for the prediction of broad-spectrum antiparasitic agents. Exp. Parasitol. 87, 194-202 (1997).

12. Thomson, S. et al. Characterisation of sterol biosynthesis and validation of $14 \alpha$-demethylase as a drug target in Acanthamoeba. Sci. Rep. 7, 8247 (2017).

13. Debnath, A. et al. CYP51 is an essential drug target for the treatment of primary amoebic meningoencephalitis (PAM). PLoS Negl. Trop. Dis. 11, e0006104 (2017).

14. Kidane, M. E. et al. Sterol methyltransferase a target for anti-amoeba therapy: Towards transition state analog and suicide substrate drug design. J. Lipid Res. 58, 2310-2323 (2017).

15. Milanes, J. E. et al. Enzymatic and structural characterization of the Naegleria fowleri glucokinase. Antimicrob. Agents Chemother. 63, e02410-18 (2019).

16. Rice, C. A. et al. Structural and functional studies of histidine biosynthesis in Acanthamoeba spp. demonstrates a novel molecular arrangement and target for antimicrobials. PLoS ONE 13, e0198827 (2018).

17. Henriquez, F. L. et al. The Acanthamoeba shikimate pathway has a unique molecular arrangement and is essential for aromatic amino acid biosynthesis. Protist 166, 93-105 (2015).

18. Greninger, A. L. et al. Clinical metagenomic identification of Balamuthia mandrillaris encephalitis and assembly of the draft genome: the continuing case for reference genome sequencing. Genome Med. 7, 113 (2015).

19. Detering, H. et al. First draft genome sequence of Balamuthia mandrillaris, the causative agent of amoebic encephalitis. Genome Announc. 3, e01013-15 (2015).

20. Myler, P. J. et al. The Seattle Structural Genomics Center for infectious disease (SSGCID). Infect. Disord. Drug Targets 9, 493-506 (2009).

21. Gilbert, D. G. Genes of the pig, Sus scrofa, reconstructed with EvidentialGene. PeerJ 7, e6374 (2019).

22. Clarke, M. et al. Genome of Acanthamoeba castellanii highlights extensive lateral gene transfer and early evolution of tyrosine kinase signaling. Genome Biol. 14, R11 (2013).

23. Shabardina, V., Kischka, T., Kmita, H., Suzuki, Y. \& Makałowski, W. Environmental adaptation of Acanthamoeba castellanii and Entamoeba histolytica at genome level as seen by comparative genomic analysis. Int. J. Biol. Sci. 14, 306-320 (2018).

24. Waterhouse, R. M. et al. BUSCO applications from quality assessments to gene prediction and phylogenomics. Mol. Biol. Evol. https://doi.org/10.1093/molbev/msx319 (2017).

25. Somervuo, P. \& Holm, L. SANSparallel: Interactive homology search against Uniprot. Nucleic Acids Res. 43, W24-29 (2015).

26. Stroehlein, A. J., Young, N. D. \& Gasser, R. B. Improved strategy for the curation and classification of kinases, with broad applicability to other eukaryotic protein groups. Sci. Rep. 8, 6808 (2018).

27. Di Tommaso, P. et al. T-Coffee: A web server for the multiple sequence alignment of protein and RNA sequences using structural information and homology extension. Nucleic Acids Res. 39, W13-17 (2011).

28. Schuster, F. L. \& Visvesvara, G. S. Efficacy of novel antimicrobials against clinical isolates of opportunistic amebas. J. Eukaryot. Microbiol. 45, 612-618 (1998).

29. McGhee, P. et al. In vitro activity of CEM-101 against Streptococcus pneumoniae and Streptococcus pyogenes with defined macrolide resistance mechanisms. Antimicrob. Agents Chemother. 54, 230-238 (2010).

30. Hamilton-Miller, J. M. Chemistry and biology of the polyene macrolide antibiotics. Bacteriol. Rev. 37, 166-196 (1973).

31. Schuster, F. L. \& Visvesvara, G. S. Axenic growth and drug sensitivity studies of Balamuthia mandrillaris, an agent of amebic meningoencephalitis in humans and other animals. J. Clin. Microbiol. 34, 385-388 (1996).

32. Laurie, M. T. et al. Functional assessment of 2,177 U.S. and international drugs identifies the quinoline nitroxoline as a potent amoebicidal agent against the pathogen Balamuthia mandrillaris. MBio 9, e02051-18 (2018).

33. Schuster, F. L., Guglielmo, B. J. \& Visvesvara, G. S. In-vitro activity of miltefosine and voriconazole on clinical isolates of free-living amebas: Balamuthia mandrillaris, Acanthamoeba spp., and Naegleria fowleri. J. Eukaryot. Microbiol. 53, 121-126 (2006).

34. Ahmad, A. F., Heaselgrave, W., Andrew, P. W. \& Kilvington, S. The in vitro efficacy of antimicrobial agents against the pathogenic free-living amoeba Balamuthia mandrillaris. J. Eukaryot. Microbiol. 60, 539-543 (2013).

35. Rice, C. A., Lares-Jiménez, L. F., Lares-Villa, F. \& Kyle, D. E. In vitro screening of the open source MMV Malaria and pathogen boxes to discover novel compounds with activity against Balamuthia mandrillaris. Antimicrob. Agents Chemother. https://doi.org/ 10.1128/AAC.02233-19 (2020). 
36. Rice, C. A., Colon, B. L., Chen, E., Hull, M. V. \& Kyle, D. E. Discovery of repurposing drug candidates for the treatment of diseases caused by pathogenic free-living amoebae. PLoS Negl. Trop. Dis. 14, e0008353 (2020).

37. Michel, R., Müller, K. D. \& Hoffmann, R. Enlarged Chlamydia-like organisms as spontaneous infection of Acanthamoeba castellanii. Parasitol. Res. 87, 248-251 (2001).

38. Sierra, S. et al. Statins as neuroprotectants: A comparative in vitro study of lipophilicity, blood-brain-barrier penetration, lowering of brain cholesterol, and decrease of neuron cell death. J. Alzheimers Dis. 23, 307-318 (2011).

39. Leimeister, C.-A. et al. Prot-SpaM: Fast alignment-free phylogeny reconstruction based on whole-proteome sequences. Gigascience 8, giy148 (2019).

40. Humann, J. L., Lee, T., Ficklin, S. \& Main, D. Structural and functional annotation of eukaryotic genomes with GenSAS. Methods Mol. Biol. 1962, 29-51 (2019).

41. Hunt, A. G. A rapid, simple, and inexpensive method for the preparation of strand-specific RNA-Seq libraries. Methods Mol. Biol. 1255, 195-207 (2015).

42. Myler, P. J., McDonald, J. A., Alcolea, P. J. \& Sur, A. Quantitative RNA analysis using RNA-Seq. Methods Mol. Biol. 1971, 95-108 (2019).

43. Bolger, A. M., Lohse, M. \& Usadel, B. Trimmomatic: A flexible trimmer for Illumina sequence data. Bioinformatics 30, 2114-2120 (2014).

44. Haas, B. J. et al. De novo transcript sequence reconstruction from RNA-seq using the Trinity platform for reference generation and analysis. Nat. Protoc. 8, 1494-1512 (2013).

45. Bushmanova, E., Antipov, D., Lapidus, A. \& Prjibelski, A. D. rnaSPAdes: A de novo transcriptome assembler and its application to RNA-Seq data. Gigascience 8, giz100 (2019).

46. Dobin, A. et al. STAR: Ultrafast universal RNA-seq aligner. Bioinformatics 29, 15-21 (2013).

47. Seppey, M., Manni, M. \& Zdobnov, E. M. BUSCO: Assessing genome assembly and annotation completeness. Methods Mol. Biol. 1962, 227-245 (2019).

48. Afgan, E. et al. Genomics virtual laboratory: A practical bioinformatics workbench for the cloud. PLoS One 10, e0140829 (2015).

49. Törönen, P., Medlar, A. \& Holm, L. PANNZER2: A rapid functional annotation web server. Nucleic Acids Res. 46, W84-W88 (2018).

50. Ye, J. et al. WEGO 2.0: a web tool for analyzing and plotting GO annotations, 2018 update. Nucleic Acids Res. 46, W71-W75 (2018).

51. Medlar, A. J., Törönen, P. \& Holm, L. AAI-profiler: Fast proteome-wide exploratory analysis reveals taxonomic identity, misclassification and contamination. Nucleic Acids Res. 46, W479-W485 (2018).

52. Xu, L. et al. OrthoVenn2: A web server for whole-genome comparison and annotation of orthologous clusters across multiple species. Nucleic Acids Res. 47, W52-W58 (2019).

53. Camacho, C. et al. BLAST+: Architecture and applications. BMC Bioinform. 10, 421 (2009).

54. Liechti, N., Schürch, N., Bruggmann, R. \& Wittwer, M. Nanopore sequencing improves the draft genome of the human pathogenic amoeba Naegleria fowleri. Sci. Rep. 9, 16040 (2019).

55. Liechti, N., Schürch, N., Bruggmann, R. \& Wittwer, M. The genome of Naegleria lovaniensis, the basis for a comparative approach to unravel pathogenicity factors of the human pathogenic amoeba N. fowleri. BMC Genom. 19, 654 (2018).

56. Kumar, S., Stecher, G., Li, M., Knyaz, C. \& Tamura, K. MEGA X: Molecular evolutionary genetics analysis across computing platforms. Mol. Biol. Evol. 35, 1547-1549 (2018).

57. Stecher, G., Tamura, K. \& Kumar, S. Molecular evolutionary genetics analysis (MEGA) for macOS. Mol. Biol. Evol. https://doi.org/ $10.1093 / \mathrm{molbev} / \mathrm{msz} 312(2020)$.

58. Stacy, R. et al. Structural genomics of infectious disease drug targets: The SSGCID. Acta Crystallogr. Sect. F. Struct. Biol. Cryst. Commun. 67, 979-984 (2011).

59. Aslanidis, C. \& de Jong, P. J. Ligation-independent cloning of PCR products (LIC-PCR). Nucleic Acids Res. 18, 6069-6074 (1990).

60. Studier, F. W. Protein production by auto-induction in high density shaking cultures. Protein Expr. Purif. 41, 207-234 (2005).

61. Ewing, B. \& Green, P. Base-calling of automated sequencer traces using phred. II. Error probabilities. Genome Res. 8, 186-194 (1998).

62. Edgar, R. C. MUSCLE: Multiple sequence alignment with high accuracy and high throughput. Nucleic Acids Res. 32, 1792-1797 (2004).

\section{Acknowledgements}

We thank Drs. Luis Fernando Lares-Jiménez \& Fernando Lares-Villa (Instituto Tecnológico de Sonora, Ciudad Obregón, Sonora, Mexico) for the pathogenic isolate of B. mandrillaris used in this study, and Aakash Sur for insightful discussions. The authors acknowledge the support of the Freiburg Galaxy Team led by Prof. Rolf Backofen, Bioinformatics, University of Freiburg, Germany funded by Collaborative Research Centre 992 Medical Epigenetics (DFG grant SFB 992/1 2012) and German Federal Ministry of Education and Research (BMBF grant 031 A538A de.NBI-RBC). Rooksana Noorai was supported by an Institutional Development Award (IDeA) from the National Institute of General Medical Sciences of the National Institutes of Health under Grant number P20GM109094. This project has been funded in part with Federal funds from the National Institute of Allergy and Infectious Diseases, National Institutes of Health, Department of Health and Human Services, under Contract No.: HHSN272201700059C and the Georgia Research Alliance (GRA) also supported this work.

\section{Author contributions}

Conceptualization, I.Q.P., C.A.R., D.E.K., and P.J.M.; methodology, I.Q.P., C.A.R., J.C., J.M., S.S. and L.T.; validation, I.Q.P. and C.A.R; formal analysis, I.Q.P., C.A.R., R.E.N., V.S., J.C. and S.S.; resources, D.E.K., W.C.V.V., J.C.M., and P.J.M.; data curation, I.Q.P. and C.A.R; writing-original draft preparation, I.Q.P. and C.A.R.; writingreview and editing I.Q.P., C.A.R., R.E.N., V.S., S.S., L.K.B., J.C.M., D.E.K., W.C.V.V., and P.J.M.; visualization, I.Q.P. and C.A.R.; supervision, I.Q.P., C.A.R., D.E.K., W.C.V.V., J.C.M., and P.J.M.; funding acquisition, D.E.K., W.C.V.V., J.C.M. and P.J.M. All authors have read and agreed to the published version of the manuscript.

\section{Competing interests}

W.C. Van Voorhis is a co-owner of ParaTheraTech, Inc, a small biotech that aims to market compounds for the therapy of parasitic diseases in Animal Health. All other authors declare no competing interests.

\section{Additional information}

Supplementary Information The online version contains supplementary material available at https://doi.org/ 10.1038/s41598-021-99903-8. 
Correspondence and requests for materials should be addressed to I.Q.P., C.A.R. or P.J.M.

Reprints and permissions information is available at www.nature.com/reprints.

Publisher's note Springer Nature remains neutral with regard to jurisdictional claims in published maps and institutional affiliations.

(c) (i) Open Access This article is licensed under a Creative Commons Attribution 4.0 International cc) License, which permits use, sharing, adaptation, distribution and reproduction in any medium or format, as long as you give appropriate credit to the original author(s) and the source, provide a link to the Creative Commons licence, and indicate if changes were made. The images or other third party material in this article are included in the article's Creative Commons licence, unless indicated otherwise in a credit line to the material. If material is not included in the article's Creative Commons licence and your intended use is not permitted by statutory regulation or exceeds the permitted use, you will need to obtain permission directly from the copyright holder. To view a copy of this licence, visit http://creativecommons.org/licenses/by/4.0/.

(C) The Author(s) 2021 